\title{
Second-order effects on the hyperfine structure of $P$ states of alkali-metal atoms
}

\author{
K. Beloy and A. Derevianko \\ Physics Department, University of Nevada, Reno, Nevada 89557
}

(Dated: November 2, 2018)

\begin{abstract}
We analyze second-order $M 1-M 1$ and $M 1-E 2$ effects to the hyperfine structure (HFS) of the lowest energy $P$ states of alkali-metal atoms arising from the coupling of the two $(J=1 / 2,3 / 2)$ fine-structure levels through the hyperfine interaction. We find these effects to be especially sizable in $\mathrm{Li}$, leading to a $9 \sigma$ correction to the most accurate reported experimental value of the $A\left(P_{1 / 2}\right)$ HFS constant of ${ }^{7} \mathrm{Li}$ [D. Das and V. Natarajan, J. Phys. B 41, 035001 (2008)]. For the remaining alkali-metal systems, the results tabulated within may be referenced as higher precision is sought in experimental determination of the HFS constants.
\end{abstract}

PACS numbers: 32.10.Fn,31.15.A-

\section{INTRODUCTION}

As experimental accuracy improves, the interpretation of measurements may require refined theoretical analysis. Measurements of the hyperfine structure (HFS) is one of such examples. The HFS of atomic systems arises from the coupling between the atomic electrons and the nuclear spin. Generally speaking, in the approximation that $J$, representing the electronic angular momentum, remains a "good" quantum number, the HFS can be accurately described by the conventional (first-order) HFS constants $A, B, C, \ldots$ [1]. Respectively, these constants describe the electronic interaction with the nuclear magnetic dipole $(M 1)$, electric quadrupole $(E 2)$, magnetic octupole (M3),.. ; these interactions are collectively referred to as the hyperfine interaction (HFI). Likewise, with $J$ being a "good" quantum number, experimental measurements of the HFS intervals, along with known values of the nuclear moments, can be used to definitively determine these HFS constants. However, for atomic states that are part of a fine-structure manifold, the nearby fine-structure levels may provide sizable contamination to the $J$-purity [2, 3, 4, [3]. In these situations, to make the connection between HFS interval measurements and HFS constants, it becomes necessary to consider the HFI coupling between fine-structure states [1]. In terms of perturbation theory, this arises in the second-order in the HFI and is dominated by the $M 1-M 1$ and $M 1-E 2$ effects.

In this paper, we provide results for calculations of the second-order corrections to the HFS for the lowest energy $P$ states of the naturally occurring alkali-metal isotopes. These isotopes, along with their respective nuclear properties, are displayed in Table [1. The HFS constants $A\left(P_{1 / 2}\right), A\left(P_{3 / 2}\right)$, and $B\left(P_{3 / 2}\right)$ have been experimentally measured for all of these isotopes (see Refs. [2, 6, 7] and references within). In a recent paper, Das and Natarajan [6] claim a significant improvement in experimental uncertainty over earlier works for most of these constants.

Due to its relatively small fine-structure splitting, the second-order effects of the HFI are most noticeable in $\mathrm{Li}$, the lightest of the alkali-metal atoms. Over thirty years ago, in experimental determination of the HFS con-
TABLE I: Nuclear properties of stable alkali-metal isotopes. $I^{\pi}$ represents the nuclear spin and parity. Nuclear dipole and quadrupole values are given in terms of nuclear magnetons $\left(\mu_{N}\right)$ and barns (b), respectively. All data is taken from Ref. [8].

\begin{tabular}{cllc}
\hline \hline Isotope & \multicolumn{1}{c}{$I^{\pi}$} & \multicolumn{1}{c}{$\mu\left(\mu_{N}\right)$} & $Q(\mathrm{~b})$ \\
\hline${ }^{6} \mathrm{Li}$ & $1^{+}$ & $0.8220473(6)$ & $-0.00082(2)$ \\
${ }^{7} \mathrm{Li}$ & $3 / 2^{-}$ & $3.2564625(4)$ & $-0.0406(8)$ \\
${ }^{23} \mathrm{Na}$ & $3 / 2^{+}$ & $2.2176556(6)$ & $0.1045(10)$ \\
${ }^{39} \mathrm{~K}$ & $3 / 2^{+}$ & $0.39150731(12)$ & $0.0585(6)$ \\
${ }^{41} \mathrm{~K}$ & $3 / 2^{+}$ & $0.21489274(12)$ & $0.0711(7)$ \\
${ }^{85} \mathrm{Rb}$ & $5 / 2^{-}$ & $1.3533515(8)$ & $0.277(1)$ \\
${ }^{87} \mathrm{Rb}$ & $3 / 2^{-}$ & $2.751818(2)$ & $0.134(1)$ \\
${ }^{133} \mathrm{Cs}$ & $7 / 2^{+}$ & $2.5829128(15)$ & $-0.00355(4)$ \\
\hline \hline
\end{tabular}

stants, Orth et al. 2, 3] recognized the need to consider the mixing of the fine-structure $P$ states of Li. However, more recent measurements of the ${ }^{6,7} \mathrm{Li} A\left(P_{1 / 2}\right)$ constants [6, 9] have overlooked this important effect. Namely, we find that the second-order correction to the ${ }^{7} \mathrm{Li} A\left(P_{1 / 2}\right)$ constant causes a shift which is a full order of magnitude more than claimed uncertainty of Das and Natarajan [6]. For the remaining alkali-metal isotopes, we find that the second-order effects are not significant at the current levels of experimental precision for the HFS constants. However as higher precision is sought, it may become necessary to include these effects. The compilation of second-order corrections provided herein may provide useful complementary data for future highprecision experiments on these isotopes.

This paper is organized as follows. In Section III, we present pertinent equations for the HFI and corresponding first- and second-order energies obtained from a standard perturbation theory analysis. In Section III we provide a reformulated tensorial analysis of the perturbation theory; this formalism provides more insight into the rotational symmetries of the various second-order contributions. We compile HFS expressions for the $P$ states of the alkali-metal atoms in Section IV. In Section V we tabulate our results, followed by concluding remarks in Section VI. Furthermore, we include an Appendix 
containing notations and expressions for spherical tensor operators appearing in Sections [I] and [II

\section{THE HYPERFINE INTERACTION}

In this Section, we recapitulate the basic properties of the hyperfine interaction and the application of perturbation theory to analyze its effects on atomic structure. This Section is an abbreviated review of the presentation provided in Ref. [4]. We employ the notation $\left\langle T_{k}\right\rangle_{I}$ to represent the expectation value of the zerocomponent operator of spherical tensor (of rank $k$ ) $T_{k}$ in the "stretched" state $\left|I, M_{I}=I\right\rangle$. This is related to the reduced matrix element through the expression

$$
\left\langle T_{k}\right\rangle_{I}=\left(\begin{array}{ccc}
I & k & I \\
-I & 0 & I
\end{array}\right)\left\langle I\left\|T_{k}\right\| I\right\rangle
$$

The hyperfine interaction can be expressed as a sum over scalar products of spherical tensors:

$$
H_{\mathrm{HFI}}=\sum_{k} T_{k}^{n} \cdot T_{k}^{e}
$$

where $T_{k}^{n}$ and $T_{k}^{e}$ are spherical tensors of rank $k(k>0)$ acting in the nuclear and electronic spaces, respectively. As $H_{\mathrm{HFI}}$ is a scalar operator in the combined space, it is convenient to work in the conventional basis formed by coupling nuclear, $\left|I M_{I}\right\rangle$, and electronic, $\left|\gamma J M_{J}\right\rangle$, states,

$$
\left|\gamma I J F M_{F}\right\rangle=\sum_{M_{J}, M_{I}} C_{I M_{I} ; J M_{J}}^{F M_{F}}\left|I M_{I}\right\rangle\left|\gamma J M_{J}\right\rangle
$$

with $\gamma$ encapsulating remaining electronic quantum numbers and the coupling coefficients being the ClebschGordon coefficients. A matrix element of the HFI between these basis states is given by

$$
\left\langle\gamma^{\prime} I J^{\prime} F^{\prime} M_{F}^{\prime}\left|H_{H F I}\right| \gamma I J F M_{F}\right\rangle=\delta_{F^{\prime} F} \delta_{M_{F}^{\prime} M_{F}}(-1)^{I+J^{\prime}+F} \sum_{k}\left\{\begin{array}{ccc}
I & J & F \\
J^{\prime} & I & k
\end{array}\right\}\left\langle I\left\|T_{k}^{n}\right\| I\right\rangle\left\langle\gamma^{\prime} J^{\prime}\left\|T_{k}^{e}\right\| \gamma J\right\rangle
$$

First-order and second-order energy corrections to the state described by electronic quantum numbers $\gamma$ and $J$ are

$$
\begin{aligned}
W_{F}^{(1)} & =\left\langle\gamma I J F M_{F}\left|H_{H F I}\right| \gamma I J F M_{F}\right\rangle=(-1)^{I+J+F} \sum_{k}\left\{\begin{array}{ccc}
I & J & F \\
J & I & k
\end{array}\right\}\left\langle I\left\|T_{k}^{n}\right\| I\right\rangle\left\langle\gamma J\left\|T_{k}^{e}\right\| \gamma J\right\rangle, \\
W_{F}^{(2)} & =\sum_{\gamma^{\prime} J^{\prime}} \frac{\left\langle\gamma I J F M_{F}\left|H_{H F I}\right| \gamma^{\prime} I J^{\prime} F M_{F}\right\rangle\left\langle\gamma^{\prime} I J^{\prime} F M_{F}\left|H_{H F I}\right| \gamma I J F M_{F}\right\rangle}{E_{\gamma J}-E_{\gamma^{\prime} J^{\prime}}} \\
& =\sum_{\gamma^{\prime} J^{\prime}} \frac{(-1)^{J-J^{\prime}}}{E_{\gamma J}-E_{\gamma^{\prime} J^{\prime}}} \sum_{k_{1}, k_{2}}\left\{\begin{array}{ccc}
I & J & F \\
J^{\prime} & I & k_{1}
\end{array}\right\}\left\{\begin{array}{ccc}
I & J & F \\
J^{\prime} & I & k_{2}
\end{array}\right\}\left\langle I\left\|T_{k_{1}}^{n}\right\| I\right\rangle\left\langle I\left\|T_{k_{2}}^{n}\right\| I\right\rangle\left\langle\gamma J\left\|T_{k_{1}}^{e}\right\| \gamma^{\prime} J^{\prime}\right\rangle\left\langle\gamma^{\prime} J^{\prime}\left\|T_{k_{2}}^{e}\right\| \gamma J\right\rangle .
\end{aligned}
$$

Here the summations exclude the case $\left(\gamma^{\prime} J^{\prime}\right)=(\gamma J)$; this will be implicit in similar summations to follow. We note here that, due to large energies associated with nuclear excitations, we treat the nuclear states as "good" quantum states. The reduced matrix elements $\left\langle I|| T_{k}^{n} \| I\right\rangle$ appearing here are associated with the nuclear moments. Specifically, the magnetic dipole $(k=1)$, electric quadrupole $(k=2)$, and magnetic octupole $(k=3)$ are given by

$$
\begin{aligned}
& \mu=\left\langle T_{1}^{n}\right\rangle_{I}, \\
& Q=2\left\langle T_{2}^{n}\right\rangle_{I}, \\
& \Omega=-\left\langle T_{3}^{n}\right\rangle_{I} .
\end{aligned}
$$

Furthermore the product of reduced matrix elements $\left\langle I|| T_{k}^{n} \| I\right\rangle\left\langle\gamma J\left\|T_{k}^{e}\right\| \gamma J\right\rangle$ appearing in Eq. (3) correspond to the conventional first-order hyperfine constants. Specifi- cally, through the $C$ constant, these are given by

$$
\begin{aligned}
& A=\frac{1}{I J}\left\langle T_{1}^{n}\right\rangle_{I}\left\langle T_{1}^{e}\right\rangle_{J}=\frac{1}{I J} \mu\left\langle T_{1}^{e}\right\rangle_{J}, \\
& B=4\left\langle T_{2}^{n}\right\rangle_{I}\left\langle T_{2}^{e}\right\rangle_{J}=2 Q\left\langle T_{2}^{e}\right\rangle_{J}, \\
& C=\left\langle T_{3}^{n}\right\rangle_{I}\left\langle T_{3}^{e}\right\rangle_{J}=-\Omega\left\langle T_{3}^{e}\right\rangle_{J} .
\end{aligned}
$$

For electronic states within a fine-structure manifold, the leading second-order effects are due to mixing with the nearby states within the manifold (i.e., $\gamma^{\prime}=\gamma$ ). Furthermore, these effects are dominated by the dipole-dipole $(M 1-M 1)$ and dipole-quadrupole $(M 1-E 2)$ terms. The constants $\eta$ and $\zeta$ have been used in Refs. [4, 5] to pa- 
rameterize these effects and are given by

$$
\begin{aligned}
\eta= & \mp \frac{(I+1)(2 I+1)}{I} \frac{\mu^{2}\left|\left\langle\gamma J|| T_{1}^{e} \| \gamma J \pm 1\right\rangle\right|^{2}}{E_{\gamma J}-E_{\gamma J \pm 1}}, \\
\zeta= & \mp \frac{(I+1)(2 I+1)}{I} \sqrt{\frac{2 I+3}{2 I-1}} \\
& \times \frac{\mu Q\left\langle\gamma J\left\|T_{1}^{e}\right\| \gamma J \pm 1\right\rangle\left\langle\gamma J\left\|T_{2}^{e}\right\| \gamma J \pm 1\right\rangle}{E_{\gamma J}-E_{\gamma J \pm 1}} .
\end{aligned}
$$

(If both $J \pm 1$ levels exist, it would be necessary to distinguish two independent $\eta$ 's and $\zeta$ 's.) Explicit formulas for matrix elements of the electronic tensors $T_{k}^{e}$ are given in Ref. [4].

\section{TENSORIAL ANALYSIS OF SECOND-ORDER EFFECTS}

In this section we reformulate the second-order contributions to the energy. We classify the second-order terms according to the underlying rotational symmetry in the nuclear and electronic spaces. As a result, specific second-order terms are connected to the first-order constants $A, B, C, \ldots$ It becomes clear from a physical standpoint why, for instance, the second-order constant $\eta$ cannot affect experimental determination of the $C$ constant, as proven by brute manner in Ref. [4]. However, the formalism here is also farther reaching, as it can easily be extended to other second-order terms past $\eta$ and to higher orders.

We begin with an effective Hamiltonian, $H_{\gamma J}^{\text {eff }}$, which produces the exact hyperfine energies when acting on the (unperturbed) coupled basis states, Eq. (2), i.e. $H_{\gamma J}^{\mathrm{eff}}\left|\gamma I J F M_{F}\right\rangle=W_{F}\left|\gamma I J F M_{F}\right\rangle$. We decompose this effective Hamiltonian into contributions of increasing orders of the HFI, $H_{\gamma J}^{\text {eff }}=H_{\gamma J}^{(1)}+H_{\gamma J}^{(2)}+\ldots$, with associated energy contributions given by $W_{F}^{(m)}=$ $\left\langle\gamma I J F M_{F}\left|H_{\gamma J}^{(m)}\right| \gamma I J F M_{F}\right\rangle$. From Eq. (3), we may infer that the first-order Hamiltonian takes the form $H_{\gamma J}^{(1)}=$ $H_{\mathrm{HFI}}$. Furthermore, from Eq. (4), we infer the secondorder Hamiltonian to be

$$
H_{\gamma J}^{(2)}=H_{\mathrm{HFI}} R_{\gamma J} H_{\mathrm{HFI}}=\sum_{k_{1}, k_{2}}\left(T_{k_{1}}^{n} \cdot T_{k_{1}}^{e}\right) R_{\gamma J}\left(T_{k_{2}}^{n} \cdot T_{k_{2}}^{e}\right)
$$

where the latter expression is obtained by using Eq. (11) to represent $H_{\mathrm{HFI}}$. The operator $R_{\gamma J}$ here is the resolvent operator; it acts in the electronic space and is given by the expressions

$$
\begin{aligned}
R_{\gamma J} & =\sum_{\gamma^{\prime} J^{\prime} M_{J}^{\prime}} \frac{\left|\gamma^{\prime} J^{\prime} M_{J}^{\prime}\right\rangle\left\langle\gamma^{\prime} J^{\prime} M_{J}^{\prime}\right|}{E_{\gamma J}-E_{\gamma^{\prime} J^{\prime}}} \\
& =\sum_{\gamma^{\prime} J^{\prime} F^{\prime} M_{F}^{\prime}} \frac{\left|\gamma^{\prime} I J^{\prime} F^{\prime} M_{F}^{\prime}\right\rangle\left\langle\gamma^{\prime} I J^{\prime} F^{\prime} M_{F}^{\prime}\right|}{E_{\gamma J}-E_{\gamma^{\prime} J^{\prime}}}
\end{aligned}
$$

It is important to realize that the resolvent operator behaves as a scalar operator under rotations in the electronic space.

We may recouple the tensor operators of the secondorder Hamiltonian, Eq. (8), to isolate parts acting in the nuclear and electronic spaces. The resulting expression is

$$
\begin{aligned}
H_{\gamma J}^{(2)}= & \sum_{k_{1}, k_{2}, k}(-1)^{k_{1}+k_{2}+k} \\
& \times\left\{T_{k_{1}}^{n} \otimes T_{k_{2}}^{n}\right\}_{k} \cdot\left\{T_{k_{1}}^{e} \otimes R_{\gamma J} T_{k_{2}}^{e}\right\}_{k} .
\end{aligned}
$$

(See the Appendix for notational descriptions; see Ref. [10] for general relations involving spherical tensors.) The second-order correction to the energy is given by the diagonal matrix elements of $H_{\gamma J}^{(2)}$ in the coupled basis:

$$
\begin{aligned}
W_{F}^{(2)}= & \left\langle\gamma I J F M_{F}\left|H_{\gamma J}^{(2)}\right| \gamma I J F M_{F}\right\rangle \\
= & (-1)^{I+J+F} \sum_{k_{1}, k_{2}, k}(-1)^{k_{1}+k_{2}+k}\left\{\begin{array}{ccc}
I & J & F \\
J & I & k
\end{array}\right\} \\
& \times\left\langle I\left\|\left\{T_{k_{1}}^{n} \otimes T_{k_{2}}^{n}\right\}_{k}\right\| I\right\rangle \\
& \times\left\langle\gamma J\left\|\left\{T_{k_{1}}^{e} \otimes R_{\gamma J} T_{k_{2}}^{e}\right\}_{k}\right\| \gamma J\right\rangle
\end{aligned}
$$

Comparing this to Eq. (3), we can see clearly that all $k$-dependent terms enter the first- and second-order energy expressions in an identical manner. However, because $H_{\text {HFI }}$ does not contain a monopole contribution, the index $k$ in Eq. (3) is limited by $k>0$, whereas the summation here is inclusive of the case $k=0$. Analyzing the $F$-dependent factors entering this expression, namely the phase factor and six- $j$ symbol, under the condition of $k=0$ gives

$$
(-1)^{I+J+F}\left\{\begin{array}{lll}
I & J & F \\
J & I & 0
\end{array}\right\}=\frac{1}{\sqrt{(2 I+1)(2 J+1)}},
$$

and we see that $F$-dependence is lost. Thus, the secondorder $k=0$ terms here only cause an overall shift to the HFS energy levels, and do not affect the interval spacing between levels.

If all second- and higher-order effects are negligible, then the HFS intervals are determined completely by the terms $\left\langle I\left\|T_{k}^{n}\right\| I\right\rangle\left\langle\gamma J\left\|T_{k}^{e}\right\| \gamma J\right\rangle$ appearing in Eq. (3); we mention again that these terms are related directly (see Eq. (5) ) to the conventional hyperfine constants $A$ $(k=1), B(k=2), C(k=3), \ldots$ As $T_{k}^{n}$ and $T_{k}^{e}$ are spherical tensors, the specific value of $k$ represents the underlying rotational symmetry in the nuclear and electronic spaces, respectively. Experimental measurements of the HFS interval spacings may be used to determine these constants. However, if the second-order effects cannot be neglected, then the terms $\left\langle I|| T_{k}^{n} \| I\right\rangle\left\langle\gamma J\left\|T_{k}^{e}\right\| \gamma J\right\rangle$ can no longer be determined by the intervals. The "constants" which could be determined would also depend on second-order effects:

$$
\begin{aligned}
& \left\langle I\left\|T_{k}^{n}\right\| I\right\rangle\left\langle\gamma J\left\|T_{k}^{e}\right\| \gamma J\right\rangle+\sum_{k_{1}, k_{2}}(-1)^{k_{1}+k_{2}+k} \\
& \quad \times\left\langle I\left\|\left\{T_{k_{1}}^{n} \otimes T_{k_{2}}^{n}\right\}_{k}\right\| I\right\rangle\left\langle\gamma J\left\|\left\{T_{k_{1}}^{e} \otimes R_{\gamma J} T_{k_{2}}^{e}\right\}_{k}\right\| \gamma J\right\rangle .
\end{aligned}
$$


We note that second-order dipole-dipole $\left(k_{1}=k_{2}=1\right)$ terms only affect determination of the $A$ and $B$ constants, as two rank-one tensors can only be coupled to form a tensor of rank $k \leq 2$. This agrees with the proof given in Ref. [4], in which the $C$ constant was shown not to be affected by the second-order constant $\eta$. However, here we extend this conclusion to dipole-dipole terms which mix states outside of the fine-structure manifold as well. Similar conclusions can also be drawn here; for example, second-order dipole-octupole terms do not affect the determination of the $A$ constant, as a rank-one and a rank-three tensor cannot be coupled to form a tensor of rank $k=1$.

The reduced matrix elements appearing here can be simplified, giving

$$
\begin{aligned}
& \left\langle I\left\|\left\{T_{k_{1}}^{n} \otimes T_{k_{2}}^{n}\right\}_{k}\right\| I\right\rangle=(-1)^{2 I+k} \sqrt{2 k+1} \\
& \quad \times\left\{\begin{array}{ccc}
k_{1} & k_{2} & k \\
I & I & I
\end{array}\right\}\left\langle I\left\|T_{k_{1}}^{n}\right\| I\right\rangle\left\langle I\left\|T_{k_{2}}^{n}\right\| I\right\rangle \\
& \left\langle\gamma J\left\|\left\{T_{k_{1}}^{e} \otimes R_{\gamma J} T_{k_{2}}^{e}\right\}_{k}\right\| \gamma J\right\rangle=(-1)^{2 J+k} \sqrt{2 k+1} \\
& \quad \times \sum_{\gamma^{\prime} J^{\prime}}\left\{\begin{array}{ccc}
k_{1} & k_{2} & k \\
J & J & J^{\prime}
\end{array}\right\} \frac{\left\langle\gamma J\left\|T_{k_{1}}^{e}\right\| \gamma^{\prime} J^{\prime}\right\rangle\left\langle\gamma^{\prime} J^{\prime}\left\|T_{k_{2}}^{e}\right\| \gamma J\right\rangle}{E_{\gamma J}-E_{\gamma^{\prime} J^{\prime}}} .
\end{aligned}
$$

These expressions may be used in Eq. (9), at which point the summation over $k$ may be carried out analytically by using a well-known six- $j$ sum rule; the resulting expression is identical to Eq. (41).

The tensorial analysis of second-order HFI effects presented in this Section could be applied to higher-order effects as well, with similar insights following. For example, we could find that experimental determination of the $D$ constant is not affected by third-order dipole-dipoledipole terms, due to the fact that three rank-one tensors cannot be coupled to form a composite tensor with the rotational symmetry of a rank-four tensor.

\section{EXPRESSIONS FOR P-STATES OF ALKALI METAL ATOMS}

In this Section we concern ourselves with the HFS equations of the lowest energy $P$-states of alkali-metal atoms, namely the isotopes of Table [. Specifically, we are considering the $n P_{1 / 2}$ and $n P_{3 / 2}$ states, with $n=2,3,4,5,6$ for isotopes of $\mathrm{Li}, \mathrm{Na}, \mathrm{K}, \mathrm{Rb}$, and $\mathrm{Cs}$, respectively; the specific $n$ will be implicit hereafter. As inferred from the arguments of the previous Section, to determine the (first-order) constants $A, B, C, \ldots$ by measurement of HFS intervals, it is necessary to have a knowledge of the higher-order effects. Here we assume that these higher-order effects are sufficiently described by the second-order dipole-dipole and dipolequadrupole constants $\eta$ and $\zeta$. These are defined in Eqs. (617); one convenience of these definitions is that $\eta$ and $\zeta$ are identical for both the $P_{1 / 2}$ and $P_{3 / 2}$ states (i.e., $\eta\left(P_{1 / 2}\right)=\eta\left(P_{3 / 2}\right) \equiv \eta$ and similar for $\zeta$ ). Below we compile specific expressions for the first-order constants in terms of the HFS intervals $\delta W_{F}^{(J)}=W_{F}^{(J)}-W_{F-1}^{(J)}$ and the constants $\eta$ and $\zeta$.

For ${ }^{6} \mathrm{Li}(I=1)$ we have

$$
\begin{aligned}
& A\left(P_{1 / 2}\right)=\frac{2}{3} \delta W_{3 / 2}^{\left(P_{1 / 2}\right)}+\frac{1}{36} \eta+\frac{1}{12 \sqrt{3}} \zeta \\
& A\left(P_{3 / 2}\right)=\frac{1}{6} \delta W_{3 / 2}^{\left(P_{3 / 2}\right)}+\frac{3}{10} \delta W_{5 / 2}^{\left(P_{3 / 2}\right)}+\frac{1}{72} \eta-\frac{1}{120 \sqrt{3}} \zeta \\
& B\left(P_{3 / 2}\right)=-\frac{1}{3} \delta W_{3 / 2}^{\left(P_{3 / 2}\right)}+\frac{1}{5} \delta W_{5 / 2}^{\left(P_{3 / 2}\right)}+\frac{1}{36} \eta+\frac{1}{20 \sqrt{3}} \zeta .
\end{aligned}
$$

For the isotopes ${ }^{7} \mathrm{Li},{ }^{23} \mathrm{Na},{ }^{39} \mathrm{~K},{ }^{41} \mathrm{~K}$, and ${ }^{87} \mathrm{Rb}(I=$ $3 / 2$ ) we have

$$
\begin{aligned}
A\left(P_{1 / 2}\right)= & \frac{1}{2} \delta W_{2}^{\left(P_{1 / 2}\right)}+\frac{1}{90} \eta+\frac{1}{15 \sqrt{5}} \zeta \\
A\left(P_{3 / 2}\right)= & \frac{1}{20} \delta W_{1}^{\left(P_{3 / 2}\right)}+\frac{4}{25} \delta W_{2}^{\left(P_{3 / 2}\right)}+\frac{21}{100} \delta W_{3}^{\left(P_{3 / 2}\right)} \\
& +\frac{1}{180} \eta-\frac{1}{150 \sqrt{5}} \zeta \\
B\left(P_{3 / 2}\right)= & -\frac{1}{4} \delta W_{1}^{\left(P_{3 / 2}\right)}-\frac{2}{5} \delta W_{2}^{\left(P_{3 / 2}\right)}+\frac{7}{20} \delta W_{3}^{\left(P_{3 / 2}\right)} \\
& +\frac{1}{30} \eta+\frac{1}{20 \sqrt{5}} \zeta, \\
C\left(P_{3 / 2}\right)= & -\frac{1}{80} \delta W_{1}^{\left(P_{3 / 2}\right)}-\frac{1}{100} \delta W_{2}^{\left(P_{3 / 2}\right)}+\frac{1}{400} \delta W_{3}^{\left(P_{3 / 2}\right)} \\
& +\frac{1}{400 \sqrt{5}} \zeta .
\end{aligned}
$$

For ${ }^{85} \mathrm{Rb}(I=5 / 2)$ we have

$$
\begin{aligned}
A\left(P_{1 / 2}\right)= & \frac{1}{3} \delta W_{3}^{\left(P_{1 / 2}\right)}+\frac{1}{315} \eta+\frac{8}{105 \sqrt{30}} \zeta \\
A\left(P_{3 / 2}\right)= & \frac{3}{50} \delta W_{2}^{\left(P_{3 / 2}\right)}+\frac{64}{525} \delta W_{3}^{\left(P_{3 / 2}\right)}+\frac{9}{70} \delta W_{4}^{\left(P_{3 / 2}\right)} \\
& +\frac{1}{630} \eta-\frac{4}{525 \sqrt{30}} \zeta, \\
B\left(P_{3 / 2}\right)= & -\frac{1}{2} \delta W_{2}^{\left(P_{3 / 2}\right)}-\frac{8}{21} \delta W_{3}^{\left(P_{3 / 2}\right)}+\frac{15}{28} \delta W_{4}^{\left(P_{3 / 2}\right)} \\
& +\frac{2}{63} \eta+\frac{1}{14 \sqrt{30}} \zeta, \\
C\left(P_{3 / 2}\right)= & \frac{1}{40} \delta W_{2}^{\left(P_{3 / 2}\right)}-\frac{1}{35} \delta W_{3}^{\left(P_{3 / 2}\right)}+\frac{1}{112} \delta W_{4}^{\left(P_{3 / 2}\right)} \\
& +\frac{3}{280 \sqrt{30}} \zeta .
\end{aligned}
$$


Finally, for ${ }^{133} \mathrm{Cs}(I=7 / 2)$ we have

$$
\begin{aligned}
A\left(P_{1 / 2}\right)= & \frac{1}{4} \delta W_{4}^{\left(P_{1 / 2}\right)}+\frac{1}{756} \eta+\frac{1}{126} \zeta \\
A\left(P_{3 / 2}\right)= & \frac{3}{56} \delta W_{3}^{\left(P_{3 / 2}\right)}+\frac{2}{21} \delta W_{4}^{\left(P_{3 / 2}\right)}+\frac{11}{120} \delta W_{5}^{\left(P_{3 / 2}\right)} \\
& +\frac{1}{1512} \eta-\frac{1}{1260} \zeta, \\
B\left(P_{3 / 2}\right)= & -\frac{5}{8} \delta W_{3}^{\left(P_{3 / 2}\right)}-\frac{1}{3} \delta W_{4}^{\left(P_{3 / 2}\right)}+\frac{77}{120} \delta W_{5}^{\left(P_{3 / 2}\right)} \\
& +\frac{1}{36} \eta+\frac{1}{120} \zeta, \\
C\left(P_{3 / 2}\right)= & \frac{1}{32} \delta W_{3}^{\left(P_{3 / 2}\right)}-\frac{1}{24} \delta W_{4}^{\left(P_{3 / 2}\right)}+\frac{7}{480} \delta W_{5}^{\left(P_{3 / 2}\right)} \\
& +\frac{1}{480} \zeta .
\end{aligned}
$$

We note that in all of the above cases, the $\eta(\zeta)$ contribution to $A\left(P_{3 / 2}\right)$ is suppressed by a factor of $1 / 2$ $(-1 / 10)$ compared to its contribution to $A\left(P_{1 / 2}\right)$.

\section{RESULTS}

In order to calculate the second-order constants $\eta$ and $\zeta$ from Eqs. (6]7), we must first generate the $P_{1 / 2}$ and $P_{3 / 2}$ electronic states. This involves solving the electron correlation problem. We employ an ab initio relativistic coupled-cluster method which includes single, double, and triple excitations from the lowest-order DiracHartree-Fock state. While both core and valence single and double excitations are included, the triple excitations involve simultaneous excitation of the valence electron with two core electrons. In other words, the triple core excitations are not incorporated in the manybody wavefunction. We refer to this method as the CCSDvT method. The most sophisticated approximation within the CCSDvT method is described in our previous work [11] for three-electron Li. For heavier alkalis (beyond Li) the approximation of Ref. [11] becomes computationally expensive and we only keep the lowest-order terms on the right-hand-side of the valence triples equation $\left(T_{v}\left[D_{v}\right]\right.$ and $T_{v}\left[D_{c}\right]$ terms in notation of Ref. [11]). For the electronic reduced matrix elements entering Eqs. (6 7), this method gives results with accuracies better than $0.1 \%$ for the lightest $(\mathrm{Li})$ and better than a few percent for the heaviest (Cs) alkali-metal system.

Table II displays our computed values of the reduced matrix elements of the electronic tensors $T_{1}^{e}$ and $T_{2}^{e}$ between $P$ states, along with experimental values for the fine structure energy splitting. The variation in these values for different isotopes of the same atomic system is below the level of precision shown. Combining these values with the nuclear data for each isotope, Table 【. we obtain the second-order constants $\eta$ and $\zeta$. These are displayed in Table [II. Also displayed in this Table are the "corrections" to the HFS constants $A\left(P_{1 / 2}\right)$,
$A\left(P_{3 / 2}\right), B\left(P_{3 / 2}\right)$, and $C\left(P_{3 / 2}\right)$ deduced from $\eta$ and $\zeta$. Here each correction is regarded as the difference between the actual constant and the measured constant based only on first-order equations. These can be inferred from the equations in Section IV e.g., for ${ }^{133} \mathrm{Cs}$ : $\Delta A\left(P_{1 / 2}\right)=(1 / 756) \eta+(1 / 126) \zeta$.

TABLE II: Computed reduced matrix elements of the electronic tensors $T_{1}^{e}$ and $T_{2}^{e}$ between $P$ states and experimental fine structure intervals $\Delta E_{\mathrm{fs}} \equiv E_{P_{3 / 2}}-E_{P_{1 / 2}}$; fine structure intervals are taken from Ref. [12].

\begin{tabular}{cccc}
\hline \hline Atom & $\begin{array}{c}\left\langle P_{3 / 2}|| T_{1}^{e}|| P_{1 / 2}\right\rangle \\
\left(\mathrm{MHz} / \mu_{N}\right)\end{array}$ & $\begin{array}{c}\left\langle P_{3 / 2}\left\|T_{2}^{e}\right\| P_{1 / 2}\right\rangle \\
(\mathrm{MHz} / \mathrm{b})\end{array}$ & $\begin{array}{c}\Delta E_{\mathrm{fs}} \\
\left(\mathrm{cm}^{-1}\right)\end{array}$ \\
\hline $\mathrm{Li}$ & 19.0 & 11.9 & 0.3366 \\
$\mathrm{Na}$ & 12.1 & 59.4 & 17.1963 \\
$\mathrm{~K}$ & -12.6 & -103 & 57.600 \\
$\mathrm{Rb}$ & 20.1 & 213 & 237.598 \\
$\mathrm{Cs}$ & 29.2 & 313 & 554.11 \\
\hline \hline
\end{tabular}

The most accurate reported value of the ${ }^{7} \mathrm{Li} A\left(P_{1 / 2}\right)$ constant based on experimental measurement of HFS intervals is that of Das and Natarajan, Ref. [6], wherein they give a value $A\left(P_{1 / 2}\right)=46.024(3) \mathrm{MHz}$. However, this value is based on first-order HFS equations and is thus completely negligent of higher-order effects. From Table III, we see that the second-order effects would cause a sizable shift of $\Delta A\left(P_{1 / 2}\right)=27.0 \mathrm{kHz}$ to this value. This shift is a full order of magnitude larger than the claimed accuracy $(3 \mathrm{kHz})$ of the constant itself. Das and Natarajan also claim accurate results for $A$ and $B$ constants for the $P$ states of all isotopes in Table $\llbracket$ with the exception of the ${ }^{6,7} \mathrm{Li} P_{3 / 2}$ and ${ }^{41} \mathrm{~K} P_{1 / 2,3 / 2}$ states.

Figure 1 displays various reported values of the ${ }^{7} \mathrm{Li}$ $A\left(P_{1 / 2}\right)$ constant and their associated error bars. Along with the value from Das and Natarajan, the Figure includes experimental values from Orth et al. 3] and Walls et al. 9]. The earlier value of Orth et al. accounted for second-order effects, whereas that of Walls et al. did not; both claim an accuracy of the same order as our computed $\Delta A\left(P_{1 / 2}\right)$. Also included in this Figure are theoretical values recently reported by our group [11] and Yerokhin [13]. This Figure also displays "corrected" values using our computed $\Delta A\left(P_{1 / 2}\right)$ for the applicable cases of Das and Natarajan [6] and Walls et al. [9]. We note that this correction causes these two values to shift farther away from the values of Orth et al. [3], Derevianko et al. [11], and Yerokhin [13]; we do not attempt to explain the resulting large discrepancy.

For the remaining HFS constants of Li, the secondorder correction again proves to be sizable, though not as pronounced as in the ${ }^{7} \mathrm{Li} A\left(P_{1 / 2}\right)$ case. For the ${ }^{6} \mathrm{Li}$ $A\left(P_{1 / 2}\right)$ constant, Das and Natarajan claim an accuracy of the same order of magnitude as our predicted secondorder correction $\Delta A\left(P_{1 / 2}\right)=4.01 \mathrm{kHz}$.

Orth et al. 22, 3] performed HFS measurements of ${ }^{6,7} \mathrm{Li}$ in the presence of strong fields and used a complex fitting scheme to obtain the HFS constants. In addition 
TABLE III: Second-order constants $\eta$ and $\zeta$ and corresponding second-order corrections to the HFS constants. All values are in $\mathrm{kHz} . x[y]$ denotes $x \times 10^{y}$.

\begin{tabular}{clccccr}
\hline \hline Isotope & \multicolumn{1}{c}{$\zeta$} & \multicolumn{1}{c}{$\Delta A\left(P_{1 / 2}\right)$} & $\Delta A\left(P_{3 / 2}\right)$ & $\Delta B\left(P_{3 / 2}\right)$ & $\Delta C\left(P_{3 / 2}\right)$ \\
\hline${ }^{6} \mathrm{Li}$ & $1.45[+2]$ & $-2.02[-1]$ & 4.01 & 2.01 & 4.02 & \\
${ }^{7} \mathrm{Li}$ & $2.53[+3]$ & $-3.41[+1]$ & $2.70[+1]$ & $1.41[+1]$ & $8.34[+1]$ & $-3.82[-2]$ \\
${ }^{23} \mathrm{Na}$ & 9.25 & 3.72 & $2.14[-1]$ & $4.03[-2]$ & $3.92[-1]$ & $4.16[-3]$ \\
${ }^{39} \mathrm{~K}$ & $9.33[-2]$ & $1.98[-1]$ & $6.94[-3]$ & $-7.20[-5]$ & $7.54[-3]$ & $2.21[-4]$ \\
${ }^{41} \mathrm{~K}$ & $2.81[-2]$ & $1.32[-1]$ & $4.25[-3]$ & $-2.38[-4]$ & $3.89[-3]$ & $1.48[-4]$ \\
${ }^{85} \mathrm{Rb}$ & $8.76[-1]$ & 2.68 & $4.01[-2]$ & $-2.34[-3]$ & $6.28[-2]$ & $5.25[-3]$ \\
${ }^{87} \mathrm{Rb}$ & 2.87 & 2.57 & $1.08[-1]$ & $8.32[-3]$ & $1.53[-1]$ & $2.87[-3]$ \\
${ }^{133} \mathrm{Cs}$ & 3.51 & $-6.69[-2]$ & $4.11[-3]$ & $2.38[-3]$ & $9.70[-2]$ & $-1.39[-4]$ \\
\hline \hline
\end{tabular}

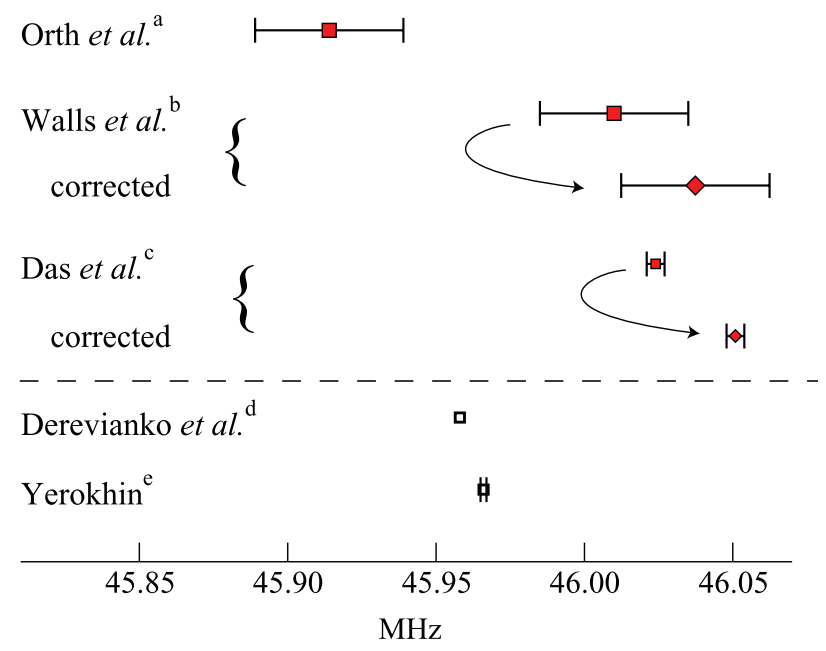

FIG. 1: (Color online) Values of the HFS constant $A\left(P_{1 / 2}\right)$ for ${ }^{7} \mathrm{Li}$. The heavy (hollow) squares denote experimental (theoretical) values reported in the respective reference, and the heavy diamonds denote the associated corrected values (of the immediately preceding value) using second-order correction $\Delta A\left(P_{1 / 2}\right)=27.0 \mathrm{kHz}$ from Table III when applicable. The uncertainty of $\Delta A\left(P_{1 / 2}\right)$ is negligible compared to the experimental uncertainties in these cases. No uncertainty is given for $d$. ${ }^{\mathrm{a}}$ Ref. [3]; ${ }^{\mathrm{b}}$ Ref. [9] ; ${ }^{\mathrm{c}}$ Ref. [6]; ${ }^{\mathrm{d}}$ Ref. [11]; ${ }^{\mathrm{e}}$ Ref. [13].

to the traditional constants, this scheme also yielded an off-diagonal constant $A\left(P_{3 / 2}, P_{1 / 2}\right)$. This constant can be related to the off-diagonal matrix element of the $T_{1}^{e}$ tensor by

$$
A\left(P_{3 / 2}, P_{1 / 2}\right)=\frac{1}{\sqrt{12}}\left|\frac{\mu}{I}\left\langle P_{3 / 2}|| T_{1}^{e}|| P_{1 / 2}\right\rangle\right| .
$$

This constant, along with the fine structure interval, parameterizes the mixing between the two fine structure levels. In Table IV] we compare our value of this offdiagonal constant for ${ }^{7} \mathrm{Li}$ (deduced from the matrix element of Table II) with the non-relativistic value from Ref. 14] and experimental value reported by Orth et al. [3]. Our (relativistic) value is in close agreement with both values.

The second-order dipole-dipole correction was considered for ${ }^{133} \mathrm{Cs}$ in Ref. [15], where a theoretical value of
TABLE IV: Computed off-diagonal constant $A\left(P_{3 / 2}, P_{1 / 2}\right)$ for ${ }^{7} \mathrm{Li}$ compared to non-relativistic and experimental values. Units are $\mathrm{MHz}$.

\begin{tabular}{lc}
\hline \hline & $A\left(P_{3 / 2}, P_{1 / 2}\right)$ \\
\hline CCSDvT ab initio; this work & 11.9 \\
non-relativ. ab initio; Ref. [14] & 11.87853 \\
experiment; Ref. [3] & $11.823(81)$ \\
\hline \hline
\end{tabular}

$\eta=22.46 \mathrm{kHz}$ was used [17]. In a following paper, Ref. [16], this effect was reevaluated using a more sophisticated third-order approach, resulting in a value of $\eta=2.09 \mathrm{kHz}$. Our present value $(\eta=3.51 \mathrm{kHz})$, based on the more complete CCSDvT method, is $68 \%$ larger than the latter value.

For the heavier alkali-metal atoms past $\mathrm{Li}$, all of the predicted second-order corrections (Table III) are one to six orders of magnitude smaller than the uncertainty in the most precise reported experimental value of the respective HFS constant. Thus, at the current levels of precision, the neglect of second-order effects in these heavier systems is acceptable. However, as experimental techniques improve and higher precision is sought, it may become necessary to consider these higher-order effects.

\section{DISCUSSION}

In this paper, we have demonstrated for the $P$ states of the alkali-metal atoms that the second-order effects cannot simply be neglected in deducing HFS constants from high-precision measurements of the HFS intervals. This is exemplified by the ${ }^{7} \mathrm{Li} A\left(P_{1 / 2}\right)$ constant, where the second-order effects are shown to cause a $9 \sigma$ shift to a recently reported value. Furthermore, we have compiled values for the leading second-order HFS effects caused by the dipole-dipole constants $\eta$ and the dipole-quadrupole constants $\zeta$ for all naturally occurring alkali-metal isotopes. These values can be used in conjunction with high-precision measurements of the HFS intervals of the alkali-metal atoms to determine the HFS constants.

This work was supported in part by the National Science Foundation. 


\section{APPENDIX A: COUPLING OF SPHERICAL TENSORS: NOTATIONS AND DEFINITIONS}

Here we provide a description for the notations used in Sections II and III along with basic definitions involving coupling of spherical tensors. For a more complete description of spherical tensors, including specific formulae for recoupling of spherical tensors and matrix elements of coupled tensor operators, see Ref. [10].

A spherical tensor of rank $k, P_{k}$, is a set of $2 k+1$ operators. We denote the individual components (operators) as $P_{k q}$, with the index $q$ taking all integer values from $-k$ to $k$. Two spherical tensors, $P_{k_{1}}$ and $Q_{k_{2}}$, may be coupled together to form a composite spherical tensor of rank $k$. The rank of the composite tensor is limited by $\left|k_{1}-k_{2}\right| \leq k \leq k_{1}+k_{2}$. We denote the coupled tensor by $\left\{P_{k_{1}} \otimes Q_{k_{2}}\right\}_{k}$; its components are given by

$$
\left\{P_{k_{1}} \otimes Q_{k_{2}}\right\}_{k q}=\sum_{q_{1} q_{2}} C_{k_{1} q_{1} ; k_{2} q_{2}}^{k q} P_{k_{1} q_{1}} Q_{k_{2} q_{2}},
$$

where $C_{k_{1} q_{1} ; k_{2} q_{2}}^{k q}$ represents Clebsch-Gordon coefficients. The scalar product of two tensors of equal rank is defined as

$$
\begin{aligned}
P_{k} \cdot Q_{k} & =(-1)^{k} \sqrt{2 k+1}\left\{P_{k} \otimes Q_{k}\right\}_{00} \\
& =\sum_{q}(-1)^{q} P_{k q} Q_{k-q}
\end{aligned}
$$

If one of the tensors in Eq. A1 is a scalar, the components of the coupled operator are simply

$$
\begin{aligned}
& \left\{P_{0} \otimes Q_{k}\right\}_{k q}=P_{00} Q_{k q} \\
& \left\{P_{k} \otimes Q_{0}\right\}_{k q}=P_{k q} Q_{00} .
\end{aligned}
$$

When $P(Q)$ here is understood to be a scalar, the composite tensor is then sufficiently represented by $P Q_{k}$ $\left(P_{k} Q\right)$.
[1] L. Armstrong, Jr., Theory of the Hyperfine Structure of Free Atoms (Willey-Interscience, New York, 1971).

[2] E. Arimondo, M. Inguscio, and P. Violino, Rev. Mod. Phys. 49, 31 (1977).

[3] H. Orth, H. Ackermann, and E. W. Otten, Z. Physik A 273, 221 (1975).

[4] K. Beloy, A. Derevianko, and W. R. Johnson, Phys. Rev. A 77, 012512 (2008).

[5] K. Beloy, A. Derevianko, V. A. Dzuba, G. T. Howell, B. B. Blinov, and E. N. Fortson, Phys. Rev. A 77, 052503 (2008).

[6] D. Das and V. Natarajan, J. Phys. B 41, 035001 (2008).

[7] S. Falke, E. Tiemann, C. Lisdat, H. Schnatz, and G. Grosche, Phys. Rev. A 74, 032503 (2006).

[8] N. J. Stone, At. Data Nucl. Data Tables 90, 75 (2005).

[9] J. Walls, J. J. Clarke, B. Lu, and W. A. van Wijngaarden, Eur. Phys. J. D 22, 159 (2003).

[10] D. A. Varshalovich, A. N. Moscalev, and V. K. Kher- sonsky, Quantum Theory of Angular Momentum (World Scientific, Singapore, 1988).

[11] A. Derevianko, S. Porsev, and K. Beloy, Phys. Rev. A 78, 010503 (2008).

[12] C. E. Moore, Atomic energy levels, vol. III (National Bureau of Standards, Washington, D.C., 1958).

[13] V. A. Yerokhin (2008), e-print arXiv:0805.0677v1.

[14] X. X. Guan and Z. W. Wang, Eur. Phys. J. D 2, 21 (1998).

[15] V. Gerginov, A. Derevianko, and C. E. Tanner, Phys. Rev. Lett. 91, 072501 (2003).

[16] W. R. Johnson, H. C. Ho, C. E. Tanner, and A. Derevianko, Phys. Rev. A 70, 014501 (2004).

[17] The values of $\eta$ in this paragraph were inferred from numbers within the cited reference, as $\eta$ was not explicitly introduced in these papers. 